\title{
Increasing the life of plunger and barrel assembly of high pressure fuel pumps
}

\author{
M.N. Salikhova ${ }^{*}$, V.A. Aleksandrov, V.S. Kukhar, I.P. Galchak, and A.M. Chudinov \\ Ural State Agrarian University, Yekaterinburg, Russia
}

\begin{abstract}
In order to identify the regularities of further increasing the efficiency of transport and technological machines, it is of interest to consider the issues of determining the impact of their design and technological parameters on the quality of running-in and the operability of fuel pumps. The article analyzes the influence of the initial operating modes and the lubricating medium on the quality of running-in and subsequent performance of high-pressure fuel pumps. On the basis of theoretical studies, equations were obtained to determine the unbalanced radial force acting on the plunger and fuel leaks in the plunger pair. These expressions make it possible to establish the dependence of the specific pressure in the plunger and barrel assembly both on the design and technological parameters of the pump.
\end{abstract}

\section{Introduction}

Transport and technological machines used at the enterprises of the agro-industrial complex are equipped with diesel engines. The efficiency of using diesel engines largely depends on the technical condition of the fuel equipment, which accounts for up to $50 \%$ of failures [1,2].

In this regard, high requirements are imposed on the fuel systems of diesel engines related to their efficiency, reliability, durability, ease of maintenance and repair, etc.

During operation, the parameters of the pumps often go beyond the regulated values. This necessitates periodic technical diagnostics of the high-pressure fuel pump, which consists in reproducing the high-speed and load operating modes of the pumps and measuring their main parameters (fuel consumption, power). High-pressure fuel pump malfunctions are determined by the technical condition of precision parts, in particular, plunger pairs. Therefore, studies aimed at increasing the resource of plunger pairs of fuel pumps are relevant.

Purpose of the study: Increasing the service life of plunger and barrel assembly of high pressure fuel pumps Determination of the dependences of the specific pressure in the plunger pair on the design and technological parameters of the high-pressure fuel pump.

Object of research: interfaces in the high-pressure fuel pump, which determine the running-in of the fuel pump as a whole.

\footnotetext{
* Corresponding author: salmar1981@mail.ru
} 


\section{Materials and Methods}

The research methodology provides for the use of the theory of probability and reliability, the use of operational tests of the high-pressure fuel pump. The theoretical research used the laws of hydraulics, mechanics and mathematical statistics.

The variety in the fuel equipment running-in modes is explained by the lack of theoretical foundations for the running-in process of precision parts and a scientifically substantiated methodology for constructing speed and load modes during the initial operation of the overhauled fuel pump.

Investigation of the running-in of all fuel pump couplings at the same time is associated with great difficulties. To avoid the arising difficulties for the study, select only those interfaces that condition the running-in of the pumps as a whole. This choice is feasible, since the nature and duration of the running-in of various couplings are not the same $[3,4,5]$.

During the pump running-in test process, the mating parts are run in: micro- and macrounevenness are smoothed out, deviations from parallelism, perpendicularity, flatness are reduced, contamination is washed out and removed. Due to this, the contact areas of the parts increase, and the specific pressures decrease to the calculated values. With the running-in process, the surface layers of the mating parts acquire optimal physicochemical properties. Along with this, the mates show malfunctions, are adjusted and tested. The absence of defects in a fully running-in pump means that all its couplings have passed the tests [6].

Since some mates are run-in easily and quickly, others are difficult and slow, it can be assumed that the pump running-in ends at the moment of the end of its testing after complete running-in of difficult-to-run mates. The shorter the period of full running-in of these mates, the earlier the pump run-in ends, since defects are detected faster, the adjustment and testing of mates occur faster. Consequently, to judge the process of complete running-in test of the pump, it is enough to investigate its hard-to-work and most critical interfaces.

Such mates in the fuel pump are the plunger pair, the discharge valve, and the tappet roller-camshaft cam mate. Let us establish for them the corresponding theoretical dependences of specific pressures on operating parameters, which will further allow us to develop optimal modes of accelerated running-in test of fuel pumps [7, 8].

To determine the specific pressure in the plunger and barrel assembly, it is necessary, first of all, to establish the unbalanced radial forces acting on the plunger.

According to the research results [9], the value of the unbalanced force for plungers $d=$ 3-15 mm reaches 2000-2500 N. Other studies have established that the calculated unbalanced force at the maximum pressure drop for pump plungers $4 \mathrm{TH}-8,5 \mathrm{xI} 0$ is $654 \mathrm{~N}$, and for the pump HK-10 - $785 \mathrm{~N}$. The study also indicates that the magnitude of the resultant radial force reaches $8000 \mathrm{~N}$ and is the main cause of local wear in the contact zone.

One of the reasons for the hydrodynamic imbalance of the plunger and barrel assembly is called the design features of the plunger pair, namely, the asymmetry of the cut-off edge on the spool part [10, 11, 12, 13, 14]. In [9], a detailed calculation of the forces pressing the plunger to the sleeve is given. The author explains the presence of radially unbalanced forces that displace the plunger in the sleeve at the moment of fuel injection by the asymmetry of the sleeve relative to the plane perpendicular to the holes. The maximum value of these forces reaches $30-50 \mathrm{~N}$.

In this work we derived [15] the reasons for the emergence of unbalanced radial forces acting on the plungers of hydraulic oil systems were established, and formulas for determining these forces. An unbalanced radial force of fluid pressure on the plunger, which creates resistance at its maximum movement, arises as a result of an uneven distribution of fluid pressure and in some cases reaches $500 \mathrm{~N}$. Consequently, unbalanced radial forces arise as a result of the fuel pressure drop, the speed of the relative movement of the rubbing surfaces, design features and inaccuracies in the manufacture of the plunger pair. The greatest 
influence on the magnitude of unbalanced radial forces and the displacement of the plunger in the sleeve is exerted by the fuel pressure drop during the active stroke of the plunger.

Since the radial unbalanced force arising from the design features can be reduced by using a plunger with two helical cut-off edges, and the unbalanced force caused by the asymmetry of the liner relative to the filling and bypass holes is insignificant, the radially unbalanced force caused by inaccuracy manufacture of parts of the plunger pair. In accordance with the technical documentation defining the geometric shape of precision pairs, ovality and taper of no more than 0.5-1 microns are allowed. The inspection found that of the number of new precision parts manufactured at the Noginsk and Yaroslavl fuel equipment plants, $60 \%$ had ovality and cone-shapedness in the range of $1-2.3$ microns.

Let us consider the case of eccentric placement of a plunger with a double-sided cone of its working surface in a bushing (Fig. 1). Because of such a cone, the cross-sectional area of the gap and the fluid pressure gradient along the length of the plunger change according to a curvilinear law.

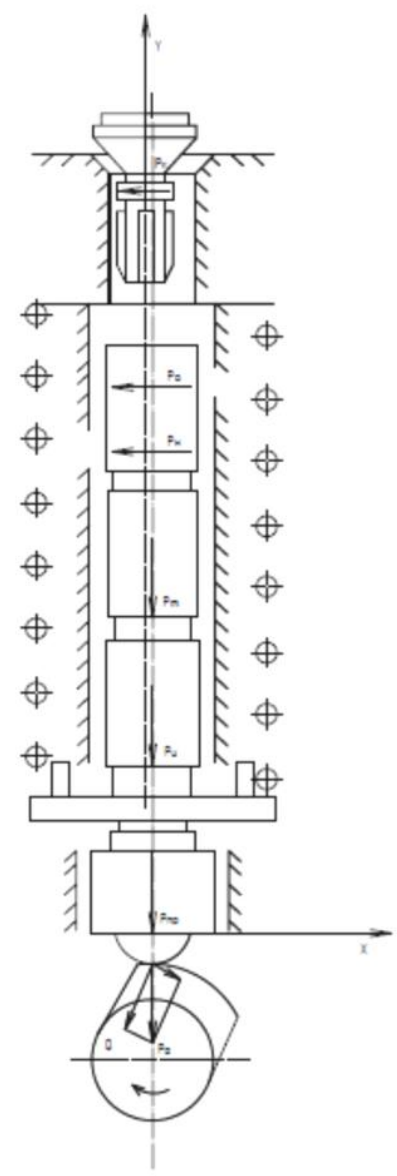

Fig. 1. Diagram of the action of forces on the details of the filling section of the fuel pump.

Plane-parallel steady motion of an incompressible fluid is described by the Navier-Stokes equations:

$$
v_{x} \frac{\partial v_{x}}{\partial x}+v_{y} \frac{\partial v_{x}}{\partial y}=v \cdot\left(\frac{\partial^{2} v_{x}}{\partial x}+\frac{\partial^{2} v_{x}}{\partial y^{2}}\right)-\frac{1}{\gamma} \frac{\partial p}{\partial x}
$$




$$
v_{x} \frac{\partial v_{y}}{\partial x}+v_{y} \frac{\partial v_{y}}{\partial y}=v \cdot\left(\frac{\partial^{2} v_{y}}{\partial x^{2}}+\frac{\partial^{2} v_{y}}{\partial y^{2}}\right)-\frac{1}{\gamma} \frac{\partial p}{\partial x}
$$

and the fluid continuity equation:

$$
\frac{\partial v_{x}}{\partial x}+\frac{\partial v_{y}}{\partial y}=0
$$

where $v_{x}$ - the projection of the fluid flow velocity on the $\mathrm{X}$-axis, $\mathrm{m} / \mathrm{s}$;

$v_{y}$ - the same on the $\mathrm{Y}$-axis, $\mathrm{m} / \mathrm{s}$;

$v$ - kinematic viscosity of the liquid, $\mathrm{m}^{2} / \mathrm{s}$;

$\gamma$ - density of liquid, $\mathrm{kg} / \mathrm{m}^{3}$;

$p$ - fluid pressure along the length of the plunger, $\mathrm{N} / \mathrm{m}^{2}$.

For a plunger and barrel assembly, we can assume $\partial v_{x}=0, v_{y}=0$. Then the NavierStokes equations and the continuity equation take the form:

$$
\begin{gathered}
\frac{\partial^{2} v_{x}}{\partial y}=\frac{1}{\mu} \frac{\partial p}{\partial x}, \\
\frac{\partial p}{\partial x}=0, \\
\frac{\partial v_{x}}{\partial x}=0,
\end{gathered}
$$

Having integrated (4) twice, we find the Poiseuille equation for the fluid flow rate in the gap of the plunger pair:

$$
v_{x}=\frac{\delta^{g}}{12 \mu} \frac{\partial p}{\partial x}
$$

where $\delta$ - clearance height, $\mathrm{m}$;

$\mu$ - dynamic viscosity of the liquid, $\mathrm{N}+\mathrm{s} / \mathrm{m}^{2}$;

$\frac{\partial p}{\partial x}$ - change in fluid pressure along the length of the gap, $\mathrm{N} / \mathrm{m}^{2}$.

After substituting (7) into (6), we obtain the Reynolds equation:

$$
\frac{\partial}{\partial x}\left(\frac{\delta^{g}}{12 \mu} \cdot \frac{\partial p}{\partial x}\right)=0
$$

which after differentiation will looks like this:

$$
\delta^{2}\left(3 \frac{\partial p}{\partial x}+\delta \frac{\delta^{2} p}{\delta x^{2}}\right)=0,
$$

By designating $\frac{\partial p}{\partial x}$ through $q$, we get:

$$
\frac{d q}{q}=-\frac{g}{\delta} d x
$$

In relation to the upper and lower parts of the spool surface, integration (10) gives:

$$
\begin{aligned}
& p_{u}=-\frac{c_{1}\left(\delta_{1}-T u x\right)^{\frac{g}{T_{u}}}+1}{3+T_{u}}+c_{2}, \\
& p_{l}=\frac{c_{3}\left(\delta_{1}-T l x\right)^{-\frac{g}{T_{l}}+1}}{T_{l}-3}+c_{4},
\end{aligned}
$$


Denoting by $l_{u}$ and $l_{1}$ the lengths of the upper and lower parts of the spool surface, $T_{u} T_{1}$ - the taper of the same parts, $\mathrm{p}_{1}, \mathrm{p}_{2}, \mathrm{p}_{3}$ - high, medium and low fluid pressure, we find for the boundary conditions:

$$
\begin{aligned}
& \delta\left|x=-l_{u}\right|=\delta_{1}-T_{u} x, p\left|x=-l_{u}\right|=p_{1}, \\
& \delta|x=0|=\delta_{1}, p|x=0|=p_{2}, \\
& \delta\left|x=l_{l}\right|=\delta_{1}+T_{l} x, p\left|x=l_{l}\right|=p_{3} .
\end{aligned}
$$

integration constants of equations (11) and (12):

$$
\begin{aligned}
& c_{1}=\frac{\left(p_{1}-p_{2}\right)\left(3+T_{u}\right)}{\delta_{1}^{\frac{3}{T_{u}}+1}-\left(\delta_{1}+T_{u} l_{u}\right)^{\frac{3}{T_{u}}+1}}, \\
& c_{2}=\frac{p_{1} \delta_{1}^{\frac{3}{T_{u}}+1}-p_{2}\left(\delta_{1}+T_{u} l_{u}\right)^{\frac{3}{T_{u}}+1}}{\delta_{1}^{\frac{3}{T_{u}}+1}-\left(\delta_{1}+T_{u} l_{u}\right)^{\frac{3}{T_{u}}+1}}, \\
& c_{3}=\frac{\left(p_{2}-p_{3}\right)\left(T_{l}-3\right)}{\delta_{1}{ }^{-\frac{3}{T_{l}}+1}-\left(\delta_{1}+T_{l} l_{l}\right)^{\frac{3}{T_{l}}+1}}, \\
& c_{4}=\frac{p_{3} \delta_{1}^{-\frac{3}{T_{l}}+1}-p_{2}\left(\delta_{1}+T_{l} l\right)^{-\frac{3}{T_{l}}+1}}{\delta_{1}^{-\frac{3}{T_{l}}+1}-\left(\delta_{1}+T_{l} l_{l}\right)^{-\frac{3}{T_{l}}+1}} .
\end{aligned}
$$

Substitute the values $\mathrm{C}_{1}$ and $\mathrm{C}_{2}$ in (11), $\mathrm{C}_{3}$ and $\mathrm{C}_{4}$ in (12):

$$
\begin{aligned}
& p_{u}=-\frac{\left(p_{1}-p_{2}\right)\left(\delta_{1}-T_{u} x\right)^{\frac{3}{T_{u}}+1}+p_{1} \delta_{1}^{\frac{3}{T_{u}}+1}-p_{2}\left(\delta_{1}+T_{u} l_{u}\right)^{\frac{3}{T_{u}}+1}}{\delta_{1}^{\frac{3}{T_{u}}+1}-\left(\delta_{1}+T_{u} l_{u}\right)^{\frac{3}{T_{u}}+1}}, \\
& p_{l}=-\frac{\left(p_{2}-p_{3}\right)\left(\delta_{1}-T_{l} x\right)^{-\frac{3}{T_{l}}+1}+p_{3} \delta_{1}-\frac{3}{T_{l}}+1}{\delta_{1}-p_{2}\left(\delta_{1}+T_{l} l_{l}\right)^{-\frac{3}{T_{l}}+1}+\left(\delta_{1}+T_{l} l_{l}\right)^{-\frac{3}{T_{l}}+1}} .
\end{aligned}
$$

Integration of (17) and (18) over the surface:

$$
p_{u}=\iint d S \int_{-l_{l}}^{0} p_{u} \cdot d x, p_{l}=\iint d S \int_{-l_{l}}^{0} p_{l} \cdot d x
$$
surface:

gives the values of the unbalanced forces for the upper and lower parts of the spool

$$
\begin{gathered}
p_{u}=\frac{\pi\left(R^{2}-r^{2}+\varepsilon^{2}\right)}{\left[(\delta-\varepsilon)^{\frac{3}{T_{u}}+1}-\left(\delta-\varepsilon+T_{u} l_{u}\right)^{\frac{3}{T_{u}}+1}\right]\left(3+2 T_{l}\right)} *\left\{\left[\left(p_{1}-p_{2}\right)(\delta-\varepsilon)^{\frac{3}{T_{u}}+2}-\right.\right. \\
\left.\left.\left(\delta-\varepsilon+T_{u} l_{u}\right)^{\frac{3}{T_{u}}+2}\right]+\left[p_{1}(\delta-\varepsilon)^{\frac{3}{T_{u}}+1}-p_{2}\left(\delta-\varepsilon+T_{u} l_{u}\right)^{\frac{3}{T_{u}}+1}\right]\left(3+2 T_{l}\right) l_{u}\right\}, \\
p_{l}=\frac{\pi\left(R^{2}-r^{2}+\varepsilon^{2}\right)}{\left[(\delta-\varepsilon)^{-\frac{3}{T_{l}}+1}-\left(\delta-\varepsilon+T_{l} l_{l}\right)^{-\frac{3}{T_{l}}+1}\right]\left(2 T_{l}-3\right)}\left\{\left[\left(p_{2}-p_{3}\right)\left(\delta-\varepsilon+T_{l} l_{l}\right)^{-\frac{3}{T_{l}}+2}-\right.\right. \\
\left.\left.(\delta-\varepsilon)^{-\frac{3}{T_{l}}+2}\right]+\left[p_{3}(\delta-\varepsilon)^{-\frac{3}{T_{u}}+1}-p_{2}\left(\delta-\varepsilon+T_{l} l_{l}\right)^{-\frac{3}{T_{l}}+1}\right]\left(2 T_{l}-3\right) l_{l}\right\}, \quad(20)
\end{gathered}
$$

$R, r$ - radius of the sleeve and plunger, $\mathrm{m}$;

$\varepsilon$ - eccentricity, $\mathrm{m}$;

$\delta$ - nominal radial clearance, $\mathrm{m}$;

The total value of the unbalanced forces will be:

$$
p=p_{u}+p_{l}
$$


To determine leaks in the plunger and barrel assembly, a number of dependencies have been proposed, of which the most interesting are the formulas of V.I.Kazartsev, V.S. Lyubenetsky with a correction by G.G. Kalish and others [16]. However, these expressions do not take into account the design and technological features of real steam plungers with their cut-off edges, filling and overflow holes.

Below are the results of a theoretical study that eliminates the noted drawback. General fuel leaks $\Delta q$, through the annular gap of the plunger pair are composed of leaks in the spool $\Delta q_{g}$ and sealing $\Delta q_{s}$ parts of the plunger pair, i.e. $\Delta q=\Delta q_{g}+\Delta q_{s}$. Bearing in mind that $\Delta q_{g}$ represents the sum of leaks through the bypass and filling holes, we get:

$$
\Delta q=\Delta q_{b}+\Delta q_{f}+\Delta q_{s}
$$

where $\Delta q$ - total fuel leakage, $\mathrm{m}^{3} / \mathrm{s}$;

$\Delta q_{b}$ - leaks through the bypass opening, $\mathrm{m}^{3} / \mathrm{s}$;

$\Delta q_{f}$ - leaks through the filling hole, $\mathrm{m}^{3} / \mathrm{s}$;

$\Delta q_{s}$ leakage through the sealing part, $\mathrm{m}^{3} / \mathrm{s}$.

With an eccentric plunger in the liner, the amount of fuel flowing through the sealing surface of the plunger is determined by the Poiseuille formula:

$$
\Delta q=\frac{\pi d_{p} \delta^{3} \Delta p \rho}{12 \mu \Delta x}
$$

Where $d_{p}$ - plunger diameter, $\mathrm{m}$;

$\delta$ - radial clearance, $\mathrm{m}$;

$\Delta p$ - pressure drop, $\mathrm{N} / \mathrm{m}^{2}$;

$\rho$ - eccentricity factor;

$\mu$ - dynamic viscosity of fuel, $\mathrm{N}^{*} \mathrm{~s} / \mathrm{m}^{2}$;

$\Delta x$ - length of the sealing surface of the plunger, $\mathrm{m}$.

Let us assume that the filling and bypass holes form a circular slot, and the spool surface of the plunger is a cylinder without a vertical groove and an oblique cut. Then the fuel leakage through the spool surface of the plunger pair according to the formula (23) will be equal to:

$$
\Delta q_{g}=\frac{\pi d_{p} \delta^{3} \Delta p \rho}{12 \mu}\left(\frac{1}{\Delta x_{1}}+\frac{1}{\Delta x_{2}}\right)
$$

However, the values revealed under this condition $\Delta q_{g}$ are far from the true ones. Therefore $\Delta q_{g}$ it is advisable to establish according to the data of its components $\Delta q_{f}$ and $\Delta q_{b}$ fuel flow method.

For determining $\Delta q_{f}$ и $\Delta q_{b}$ we divide the spool surface of the plunger along the line 00 into two parts a and c (Fig. 2). The line $0-0$ will be called the line of equal fuel currents. Taking into account the entire contour of the spool part of the plunger, we find:

$$
\begin{aligned}
\Delta q_{b} & =\frac{a \delta^{3} \Delta p \rho}{12 \mu}\left(\frac{1}{\Delta x_{b . a v}}+\frac{1}{\Delta x_{b . a v}^{\prime} \cos \alpha}\right)+\frac{b \delta^{3} \Delta p \rho}{12 \mu L_{1 a v}}, \\
\Delta q_{f} & =\frac{c \delta^{3} \Delta p \rho}{12 \mu}\left(\frac{1}{\Delta x_{f . a v}}+\frac{1}{\Delta x_{f . a v}^{\prime} \cos \alpha}\right)+\frac{H \delta^{3} \Delta p \rho}{12 \mu L_{2 a v}}
\end{aligned}
$$

where $\Delta x_{b . a v}, \Delta x_{b . a v}^{\prime}, \Delta x_{f . a v}, \Delta x_{f . a v}^{\prime}, L_{1 a v}, L_{2 a v}$ - average lengths of fuel flow lines, 


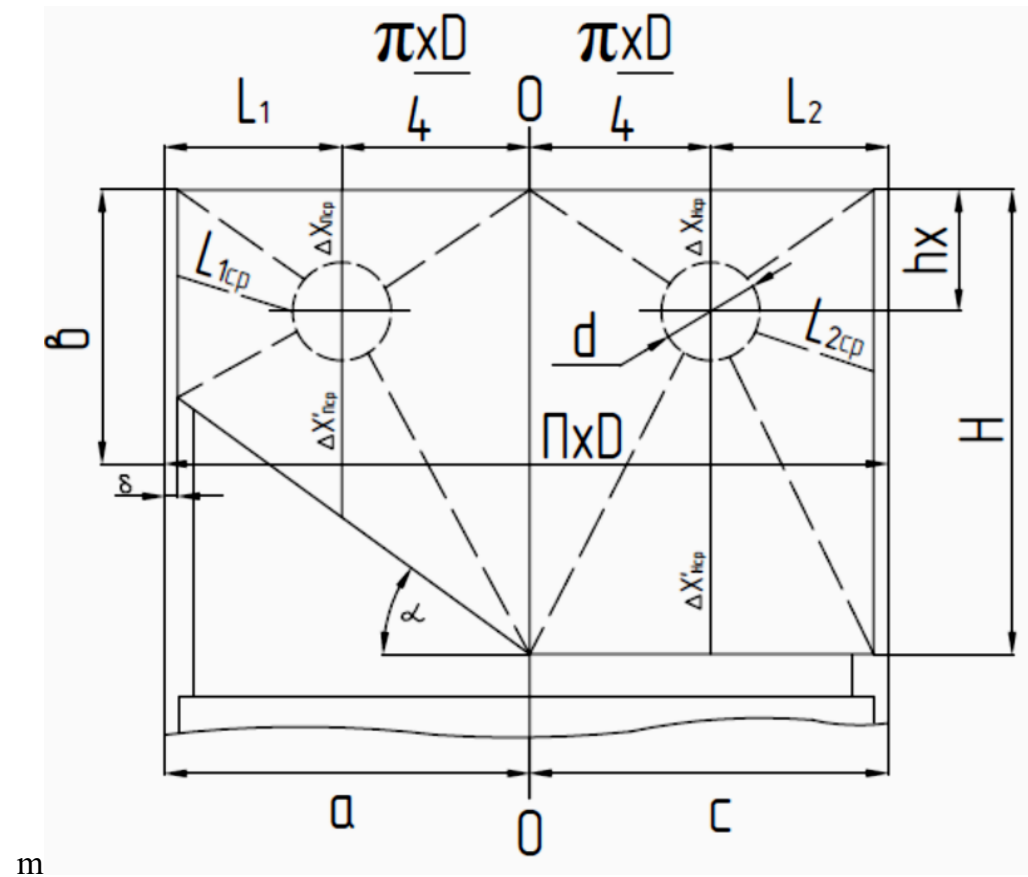

Fig. 2. Fuel flow lines in the plunger pair. be:

Then the expression to identify common fuel leaks in plunger-liner conjunction will then

$$
\Delta q=\frac{a \delta^{3} \Delta p \rho}{12 \mu}\left(\frac{1}{\Delta x_{b . a v}}+\frac{1}{\Delta x_{b . a v}^{\prime} \cos \alpha}\right)+\frac{b \delta^{3} \Delta p \rho}{12 \mu L_{1 a v}}+\frac{c \delta^{3} \Delta p \rho}{12 \mu}\left(\frac{1}{\Delta x_{f . a v}}+\frac{1}{\Delta x_{f . a v}^{\prime}}\right)+\frac{H \delta^{3} \Delta p \rho}{12 \mu L_{2 a v}}+\frac{\pi d_{p} \Delta p \rho}{12 \mu d x} .
$$

After simplifying (27), we have:

$$
\Delta q=\frac{\pi d_{p} \delta^{3} \Delta p \rho}{12 \mu}\left[\frac{1}{2}\left(\frac{1}{\Delta x_{b . a v}}+\frac{1}{\Delta x_{b . a v}^{\prime} \cos \alpha}\right)+\frac{1}{\pi d_{p} L_{1 a v}}+\frac{1}{2}\left(\frac{1}{\Delta x_{f . a v}}+\frac{1}{\Delta x_{f . a v}^{\prime}}\right)+\frac{H}{\pi d_{p} L_{2 a v}}+\frac{1}{\Delta x}\right] .
$$

Denoting the expression in square brackets through $\mathrm{M}_{k}$, we get:

$$
\Delta q=\frac{\pi d_{p} \delta^{3} \Delta p \rho M_{k}}{12 \mu}
$$
$\mathrm{m}^{-1}$.

where $M_{k}$ - value characterizing the design and technological features of plunger pairs,

For plunger and barrel assembly with one cut-off edge, the $\mathrm{M}_{k}$ value is 1.84 , and with two cut-off edges - 1.92 .

\section{Conclusion}

On the basis of research, analytical dependences were obtained to determine in the plunger pair the unbalanced radial force acting on the plunger and fuel leaks. These formulas make it possible to establish the dependence of the specific pressure in the plunger pair both on the design and technological parameters of the pump. 


\section{References}

1. V.V. Efros, Sh.V. Saidov, G.M. Kamalov, Dvigatelestroyeniye, 9, 34-36 (1989)

2. V.Yu. Matveev, A.E. Krupin, Agrotechnics and energy supply, 1, 11-20 (2016)

3. Vikas Radhakrishna Deulgaonkar, Kundan Pawar, Pratik Kudle, Atharva Raverkar, Amod Raut, Engineering Failure Analysis, 105, 1262 - 1272 (2019)

4. Yu.A. Zakharov, E.A. Kulkov, Modern scientific research and innovations, 3(2), 156$160(2015)$

5. I.I. Gabitov, A.V. Negovora, Fuel equipment of automotive diesel engines, 216 (Ufa, Publishing house BashGAU, 2004)

6. B.P. Zagorodskikh, V.P. Lyalyakin, P.A. Plotnikov, Repair and regulation of fuel equipment of autotractor and combine diesel engines, 212 (M., FGNU "Rosinformagrotech, 2006)

7. V.V. Antipov, Wear of precision parts and violation of the characteristics of diesel fuel equipment, 177 (M., Mechanical Engineering, 1972)

8. D.F. Gurevich, Mechanization and electrification of agriculture, 77, 128-134(1959)

9. M.A. Fetisov, Study of wear of plunger pairs of the YaMZ-238NB pump and increasing their durability, 24 (Sarat. Institute of mechanization of villages. households named after M. I. Kalinina, Saratov, 1970)

10. V.N. Ivanov, A.I. Ilyin, V.A. Cherenkevich, Electric and diesel traction, 8, 14-18 (1962)

11. Pavol Pecho, Martin Bugaj, Transportation Research Procedia, 35, 287 - 294 (2018)

12. L.B. Leontiev, A.L. Leontiev, Sudostroenie, 3, 40-41 (2011)

13. M.M. Tashpulatov, Ensuring the operability of diesel fuel supply equipment, 128 (Tashkent, Fan, 1990)

14. V.P. Tyukavin, Izvestiya vuzov, Forest Journal, 4 (1964)

15. T.M. Bashta, Gidroprivod i gidropneumoavtomatika, 320 (M., Mashinostroenie, 1972)

16. A.A. Mylov, Development of a method for assessing the technical condition of DTA plunger pairs during repair, 135 (RGAZU, M., 2005) 